\title{
ON $(s, S)$ INVENTORY SYSTEM WITH RANDOM LEAD TIME AND REPEATED DEMANDS
}

\author{
P. V. USHAKUMARI
}

Received 20 June 2006; Revised 23 September 2006; Accepted 8 October 2006

We consider an $(s, S)$ inventory system with random lead time and repeated demands of unsatisfied demands from the orbit. Whenever the inventory level falls to the level $s$, an order is placed to bring the level to $S$. The quantity ordered is $M=S-s$. Demands to the system are served immediately if there is a positive inventory. Otherwise it will go to a pool of unsatisfied customers called orbit. After a random amount of time, that demand is retried for service. We assume a Markovian setup for the time between consecutive arrivals, replenishments, and retrials. We obtained the condition for ergodicity of the system, steady state system size probabilities, expected length of the busy period of the system, expected inventory level, expected number of customers waiting in the orbit, expected waiting times, and so forth. A control problem is studied and some numerical illusrtations are provided.

Copyright (c) 2006 P. V. Ushakumari. This is an open access article distributed under the Creative Commons Attribution License, which permits unrestricted use, distribution, and reproduction in any medium, provided the original work is properly cited.

\section{Introduction}

The $(s, S)$ inventory system with positive lead time has been studied by several researchers. A brief account of the work carried out in this field can be seen from $[4-6,8]$, and so forth. In most of the inventory models considered so far, demanded items are directly delivered from the stock if available. The demands occurring during the stock-out period are either lost (lost sales) or satisfied only after the arrival of ordered items (backlogging). In the latter case it is assumed that either all or a prefixed number of demands occurring during the stock-out period (partial backlogging) are satisfied. See [3, 9-11] for a review.

In all these articles, the authors considered that demands to the system are either lost or served by backlogging. However, in some applications, the demands during the stock-out period go to an orbit of unsatisfied customers and after a random amount of time, retry for service. We can see such situations in production inventory systems with positive lead times. This type of retrial inventory systems has not been studied much in the literature.

Hindawi Publishing Corporation

Journal of Applied Mathematics and Stochastic Analysis

Volume 2006, Article ID 81508, Pages 1-22

DOI 10.1155/JAMSA/2006/81508 
Numerical analysis of the $(s, S)$ inventory system with repeated attempts has been studied recently in [1]. In that work the authors proceeded with an algorithmic analysis of the system. In the context of reliability, [7] studied a $k$-out-of- $n$ system with repair and retrial of failed units from the orbit. In the queuing situation, retrial queues have been studied extensively in the literature. A detailed account of the work carried out in this field can be seen in [2].

In this paper, we have considered $(s, S)(s<S)$ inventory system in which random lead time for quantities purchased are allowed. Whenever the inventory level falls to $s$, an order is placed to bring the level to $S$. The quantity ordered is $M=S-s$. The maximum inventory level $S$ is assumed to be greater than $2 s$. There is a positive lead time for the fulfillment of orders placed and the inventory level can be reduced to zero during this period. Demands to the system are served immediately if there is a positive inventory. Each demand is only for one item. If at the time of a demand the inventory level is zero (i.e., during the dry period) that demand is not satisfied, instead it goes to an orbit of unsatisfied customers. These unsatisfied demands will retry for service after a random amount of time. We assume a Markovian setup here. Interdemand times are assumed to follow an exponential distribution with rate $\lambda(>0)$. The time between consecutive replenishments is assumed to follow exponential distribution with rate $\mu(>0)$. Only one order for replenishment is pending at any given time. The time between successive retrials is also random and follows exponential distribution with rate $\alpha_{j}(>0)$ when $j$ demands in the orbit. The interarrival times, the inter-retrial times, and times between consecutive replenishments are assumed to be independent of each other.

Here we attempt to get a closed form solution of the system. We obtained the sufficient condition for ergodicity of the system. Also we obtained the steady state system size probabilities, expected length of busy period of the system, expected waiting time in the orbit, expected inventory level, expected number of waiting customers, and so forth. A control problem is studied and optimal values of the control variates $s$ and $S$ are investigated.

The paper is organized as follows. Section 2 gives the model description and in Section 3, we obtained condition for ergodicity of the system. Section 4 gives the steady state system size probabilities, marginal distributions of the inventory level and waiting customers, average inventory level, expected number of waiting customers in the orbit, and so forth. Section 5 gives the busy period analysis and Section 6 discusses waiting time distribution and the expected waiting times in the orbit. In Section 7 an optimization problem is studied and optimum values of the control variates are obtained. Some numerical illustrations are also provided in this section.

\section{Model description}

We assume that the interarrival times between the primary demands follow an exponential disrtibutiion with rate $(\lambda)$ and that between consecutive replenishments are also exponential with rate $\mu$. Also the time between consecutive retrials is exponential with rate $\alpha_{j}$ when $j$ demands in the orbit. Define $I(t)$ and $N(t)$ as the inventory level and the number of customers in the orbit at time $t$. Then the process $\{(I(t), N(t)), t \geq 0\}$ forms a bivariate Markov process over the state space $E=\{0,1,2, \ldots, s, s+1, \ldots, S\} \times\{0,1,2, \ldots\}$. 
Its infinitesimal transition rates $q_{(i, j)}$ are given by,

(1) for $s<i \leq S, j \geq 1$,

$$
q_{(i, j)(n, m)}= \begin{cases}\lambda & \text { if }(n, m)=(i-1, j), \\ j \alpha_{j} & \text { if }(n, m)=(i-1, j-1), \\ -\left(\lambda+j \alpha_{j}+\mu\right) & \text { if }(n, m)=(i, j),\end{cases}
$$

(2) for $0<i \leq s, j \geq 1$,

$$
q_{(i, j)(n, m)}= \begin{cases}\lambda & \text { if }(n, m)=(i-1, j), \\ j \alpha_{j} & \text { if }(n, m)=(i-1, j-1), \\ \mu & \text { if }(n, m)=(i+M, j), \\ -\left(\lambda+j \alpha_{j}+\mu\right) & \text { if }(n, m)=(i, j),\end{cases}
$$

(3) for $i=0, j \geq 1$,

$$
q_{(i, j)(n, m)}= \begin{cases}\mu & \text { if }(n, m)=(M, j) \\ \lambda & \text { if }(n, m)=(i, j+1) \\ -(\lambda+\mu) & \text { if }(n, m)=(i, j)\end{cases}
$$

(4) for $0<i \leq s, j=0$,

$$
q_{(i, j)(n, m)}= \begin{cases}\lambda & \text { if }(n, m)=(i-1, j), \\ \mu & \text { if }(n, m)=(i+M, j), \\ -(\lambda+\mu) & \text { if }(n, m)=(i, j),\end{cases}
$$

(5) for $i=0, j=0$,

$$
q_{(i, j)(n, m)}= \begin{cases}\mu & \text { if }(n, m)=(M, j) \\ \lambda & \text { if }(n, m)=(i, j+1) \\ -(\lambda+\mu) & \text { if }(n, m)=(i, j)\end{cases}
$$




\section{Ergodicity}

3.1. Sufficient condition for ergodicity. Sufficient condition for ergodicity of this system can be determined with the help of the criteria based on mean drifts. The following theorem [12] is most convenient.

Theorem 3.1. Let $X(t)$ be a Markov process with discrete state space $S$ and the rates of transition $q_{s p}, s, p \in S, \Sigma_{p} q_{s p}=0$. Assume that there exists

(i) a function $\phi(s), s \in S$, which is bounded below (called Lyapunov test function),

(ii) a positive number $\epsilon$ such that the variable $y_{s}=\Sigma_{p \neq s} q_{s p}(\phi(p)-\phi(s))<\infty$ for all $s$ in $S$,

(iii) $y_{s} \leq-\epsilon$ for all $s$ in $S$ except perhaps a finite number of states.

Then the process $X(t)$ is regular and ergodic.

Thus for the model under investigation, we consider the following Lyapunov function $\phi(i, j)=j-\mathrm{a} i$, where "a" is a nonnegative parameter which will be determined later. Then the mean drift $y_{i j}$ is given by

$$
y_{i j}= \begin{cases}\mathrm{a} \lambda+j \alpha_{j}(\mathrm{a}-1) & \text { if } 0<i \leq S, j \geq 1, \\ \lambda-\mathrm{a} \mu M & \text { if } i=0, j \geq 0, \\ \mathrm{a} \lambda & \text { if } s<i \leq S, j=0, \\ \mathrm{a}(\lambda-M \mu) & \text { if } 0<i \leq s, j=0 .\end{cases}
$$

Since for all $i$, there exists

$$
\lim _{j \rightarrow \infty} y_{i j}= \begin{cases}(\mathrm{a}-1) \infty & \text { if } 0<i \leq S, \\ \lambda-\mathrm{a} \mu M & \text { if } i=0, \\ \mathrm{a}(\lambda-M \mu) & \text { if } 0<i \leq s .\end{cases}
$$

The assumptions of Tweedie's theorem hold if and only if all variables are negative. That is, $a-1<0, \lambda-a \mu M<0$, and $\lambda-M \mu<0$. These conditions represent a set of linear inequalities for the unknown parameter $a$. Clearly, they can be written in the form $(\lambda / M \mu)<a<1$. Such an $a$ can be found if and only if the interval $(\lambda / M \mu, 1)$ is not empty. That is, if and only if $\lambda<M \mu$ which is the sufficient condition for ergodicity of this model. 


\section{Steady state results}

4.1. Formulation. Define $P(i, j, t)=P(I(t)=i, N(t)=j /(I(0)=S, N(0)=0),(i, j) \in E$.

The forward Kolmogorov differential difference equations associated with the Markov process are as follows:

$$
\begin{aligned}
\frac{d}{d t} P(S, j, t)= & -\left(\lambda+j \alpha_{j}\right) P(S, j, t)+\mu P(s, j, t), \quad j \geq 1, \\
\frac{d}{d t} P(i, j, t)= & -\left(\lambda+j \alpha_{j}\right) P(i, j, t)+(j+1) \alpha_{j+1} P(i+1, j+1, t)+\lambda P(i+1, j, t) \\
& +\mu P(i-M, j, t), \quad S-s \leq i \leq S-1, j \geq 1, M=S-s>s, \\
\frac{d}{d t} P(i, j, t)= & -\left(\lambda+j \alpha_{j}+\mu\left(1-\delta_{i}\right)\right) P(i, j, t)+(j+1) \alpha_{j+1} P(i+1, j+1, t) \\
& +\lambda P(i+1, j, t), \quad 0<i \leq S-s-1, j \geq 1, \\
\frac{d}{d t} P(S, 0, t)= & -\lambda P(S, 0, t)+\mu P(s, 0, t), \\
\frac{d}{d t} P(i, 0, t)= & -\lambda P(i, 0, t)+\alpha_{1} P(i+1,1, t)+\lambda P(i+1,0, t) \\
& +\mu\left(1-\delta_{i}\right) P(i-M, 0, t), \quad s<i \leq S-1, \\
& +\lambda[P(0, j-1, t)+P(1, j, t)], \quad j \geq 1, \\
& +\mu\left(1-\delta_{i}\right) P(i-M, 0, t), \quad s<i \leq S-1, \\
\frac{d}{d t} P(i, 0, t)= & \lambda P(i, 0, t)+\alpha_{1} P(i+1,1, t)+\lambda P(i+1,0, t) \\
\frac{d}{d t} P(0, j, t)= & -(\lambda+\mu) P(0, j, t)+(j+1) \alpha_{j+1} P(1, j+1, t) \\
&
\end{aligned}
$$

where

$$
\delta_{i}= \begin{cases}1 & \text { if } s+1 \leq i \leq S-s-1 \\ 0 & \text { otherwise }\end{cases}
$$

Let the initial condition be $P(S, 0,0)=1$ and $P(i, j, 0)=0$ for all $i, j$ not all zero. The above equations will be solved using Laplace transforms.

4.2. Method of solution. Denote the Laplace transform of $F(t)$ by $F(z)$. Let $P(i, j, z)=$ $\int e^{-z t} P(i, j, t) d t$ be the Laplace transform of $P(i, j, t)$. From $(4.1)$, we have

$$
\left(z+\lambda+j \alpha_{j}\right) P(S, j, z)=\mu P(i, j, z), \quad j \geq 1,
$$




$$
\begin{aligned}
& \left(z+\lambda+j \alpha_{j}\right) P(i, j, z)=(j+1) \alpha_{j+1} P(i+1, j+1, z)+\lambda P(i+1, j+1, z) \\
& +\mu P(i-M, j, z), \quad S-s \leq i \leq S-1, j \geq 1, \\
& \left(z+\lambda+\mu\left(1-\delta_{i}\right)+j \alpha_{j}\right) P(i, j, z) \\
& =(j+1) \alpha_{j+1} P(i+1, j+1, z)+\lambda P(i+1, j, z), \quad 0<i \leq S-s-1, \\
& (\lambda+z) P(S, 0, z)-P(S, 0,0)=\mu P(s, 0, z), \\
& (\lambda+z) P(i, 0, z)=\alpha_{1} P(i+1,1, z)+\lambda P(i+1,0, z) \\
& +\mu\left(1-\delta_{i}\right) P(i-M, 0, z), \quad s<i \leq S-1, \\
& (\lambda+\mu+z) P(i, 0, z)=\alpha_{1} P(i+1,1, z)+\lambda P(i+1,0, z), \quad 0<i \leq s, \\
& (\lambda+\mu+z) P(0, j, z)=\lambda[P(0, j-1, z)+P(1, j, z)] \\
& +(j+1) \alpha_{j+1} P(1, j+1, z), \quad j \geq 1 .
\end{aligned}
$$

Equation (4.3) can be used to express $P(i, j, z)$ in terms of $P(0,0, z)$ by solving $(4.3)$ as a difference equation in $i$ and $j$. Set $\alpha_{j}=\alpha / j$. Following the standard difference equation technique, set

$$
P(i, j, z)=y^{-j} P(i, 0, z)=y^{-(i+j)} P(0,0, z), \quad i, j \geq 0 .
$$

Equation (4.10) to be a solution of (4.3), we must have from (4.3),

$$
\mu y^{M}-(z+\lambda+\alpha)=0
$$

By Rouche's theorem, $(4.11)$ has $(M-1)$ roots inside and one root outside the unit circle $|y|=1$. Let the outside root be $y_{0}(z)$. Hence

$$
P(i, j, z)=\left(y_{0}(z)\right)^{-(i+j)} P(0,0, z), \quad i \geq 0, j \geq 0 .
$$

From (4.8),

$$
P(i+1,0, z)=\left[\frac{(z+\lambda+\mu) y_{0}(z)}{\alpha+\lambda y_{0}(z)}\right]^{i+1} P(0,0, z), \quad 0 \leq i \leq s .
$$


From (4.7),

$$
\begin{aligned}
& P(i+1,0, z)=\left[\frac{y_{0}(z)(z+\lambda)}{\alpha+\lambda y_{0}(z)}\right]^{i}\left[\frac{\left(\alpha+\lambda y_{0}(z)\right)(z+\lambda+\mu)}{\lambda+z}\right]^{s+1} P(0,0, z), \quad s<i \leq S-s-1, \\
& P(i+1,0, z)=\frac{y_{0}(z)}{\alpha+\lambda y_{0}(z)}\left\{\left(\frac{z+\lambda+\mu)}{z+\lambda}\right)^{s+1}(z+\lambda)\left(y_{0}(z)(z+\lambda)\right)^{i}\right. \\
& \left.-\mu\left[\frac{y_{0}(z)(z+\lambda+\mu)}{\alpha+\lambda y_{0}(z)}\right]^{i-M}\right\} P(0,0, z), \quad S-s \leq i \leq S-1, \\
& P(0, j, z)=\frac{\left(y_{0}(z)\right)^{-j}}{\lambda+\mu+z}\left(\lambda\left(1+y_{0}(z)\right)+\mu+z\right) P(0,0, z), \quad j \geq 1, \\
& P(i, j, z)=\frac{\left(y_{0}(z)\right)^{-j}(\lambda+\mu+z)}{(z+\lambda+\mu+\alpha)}\left[\frac{\lambda+\mu+z}{\alpha+\lambda y_{0}(z)}\right]^{i} P(0,0, z), \quad 0<i \leq s, j \geq 1, \\
& P(i, j, z)=\left(y_{0}(z)\right)^{i-j}(\lambda+z)^{i+1-s}\left(\alpha+\lambda y_{0}(z)\right)^{s-i} \\
& \times(\lambda+\mu+z)^{s} P(0,0, z), \quad s<i \leq S-s-1, j \geq 1, \\
& P(S-s, j, z)=\left\{\frac{\mu}{\alpha-\mu} \frac{z+\lambda+\mu}{z+\lambda+\alpha} \frac{\left(y_{0}(z)\right)^{-j}}{z+\lambda+\alpha+\mu}\left[1-\left(\frac{z+\lambda+\mu}{z+\lambda+\alpha}\right)^{s}\right]\right. \\
& \left.+\frac{\left(y_{0}(z)\right)^{-j}}{z+\lambda+\mu}\left(\lambda\left(1+y_{0}(z)\right)+\mu+z\right)\right\} P(0,0, z), \quad j \geq 1, \\
& P(S-i, j, z)=\frac{\mu}{\alpha-\mu} \frac{(z+\lambda+\mu)^{2}}{(z+\lambda+\alpha)(z+\lambda+\mu+\alpha)}\left(\frac{z+\lambda+\mu}{\alpha+\lambda y_{0}(z)}\right)^{s}\left(y_{0}(z)\right)^{-j}\left(\frac{\lambda+\alpha\left(y_{0}(z)\right)^{-1}}{z+\lambda+\alpha}\right) \\
& \times\left[1-\left(\frac{z+\lambda+\mu}{z+\lambda+\alpha}\right)^{i+1}\right] P(0,0, z), \quad 0<i \leq s-1, j \geq 1, \\
& P(S, j, z)=\frac{\mu(z+\lambda)}{z+\lambda+\alpha}\left(y_{0}(z)\right)^{s-j}(\lambda+\mu+z)^{s} P(0,0, z), \quad j \geq 1 .
\end{aligned}
$$

4.3. Steady state system size probabilities. The steady state distribution of the system size can be obtained by applying the final value theorem. Let $q(i, j)=\lim _{z \rightarrow 0} z P(i, j, z)$, $(i, j) \in E$ and $k=\lim _{z \rightarrow 0} y_{0}(z)$. Then $k$ satisfies the equation $\mu k^{M}-(\lambda+\alpha)=0$ which gives $k=((\lambda+\alpha) / \mu)^{1 / M}$. Then we have

$$
\begin{aligned}
& q(i, 0)=\left[\frac{k(\lambda+\mu)}{\alpha+k \lambda}\right]^{i} q(0,0), \quad 0<i \leq s+1, \\
& q(i, 0)=\left(\frac{k \lambda}{\alpha+k \lambda}\right)^{i-1}\left[(\alpha+k \lambda)\left(\frac{\lambda+\mu}{\lambda}\right)\right]^{s} q(0,0), \quad s+1<i \leq S-s,
\end{aligned}
$$




$$
\begin{aligned}
& q(i, 0)=\frac{k}{\alpha+k \lambda}\left\{\left(\frac{\lambda+\mu}{\lambda}\right)^{s}(k \lambda)^{i-1} \lambda-\mu\left(\frac{k \lambda+\mu}{\alpha+k \lambda}\right)^{i-1-M}\right\} q(0,0), \quad S-s+1 \leq i \leq S, \\
& q(0, j)=\frac{k^{-j}}{\lambda+\mu}(\lambda(1+k)+\mu) q(0,0), \quad j \geq 1, \\
& q(i, j)=\frac{k^{-j}(\lambda+\mu)^{2}}{(\lambda+\mu+\alpha)(k \lambda+\alpha)} q(0,0), \quad 0<i \leq s, j \geq 1, \\
& q(i, j)=k^{i-j} \lambda^{i+1-s}(\alpha+k \lambda)^{s-i}(\lambda+\mu) q(0,0), \quad s<i \leq S-s-1, j \geq 1, \\
& q(S-s, j)=\left\{\frac{\mu}{\alpha-\mu} \frac{\lambda+\mu}{\lambda+\alpha} \frac{k^{-j}}{\lambda+\mu+\alpha}\left[1-\left(\frac{\lambda+\mu}{\lambda+\alpha}\right)^{s}\right]+\frac{k^{-j}(\lambda(1+k)+\mu)}{\lambda+\mu}\right\} q(0,0), \quad j \geq 1, \\
& q(S-i, j)=\frac{\mu}{\alpha-\mu} \frac{\lambda+\mu}{\lambda+\alpha} \frac{\lambda+\mu}{\lambda+\mu+\alpha}\left(\frac{k(\lambda+\mu)}{\alpha+k \lambda}\right)^{s} k^{-j}\left(\frac{\lambda+\alpha k^{-1}}{\lambda+\alpha}\right)\left(1-\left(\frac{\lambda+\mu}{\lambda+\alpha}\right)^{i+1}\right) q(0,0), \\
& q=1,2, \ldots, s-1, j \geq 1,
\end{aligned}
$$

$q(S, j)=\frac{\lambda \mu}{\lambda+\alpha} k^{s-j}(\lambda+\mu) q(0,0), \quad j \geq 1$.

$q(0,0)$ can be obtained in such a way that $\sum_{i=0}^{S} \sum_{j=0}^{\infty} q(i, j)=1$, which gives $q(0,0)$ as $q(0,0)=\left(1+T_{1}+T_{2}+T_{3}+T_{4}\right)^{-1}$, where

$$
\begin{aligned}
T_{1}= & \frac{k(\lambda+\mu)}{\alpha+(k-1) \lambda+\mu}\left[1-\left(\frac{\lambda+\mu}{\alpha+k \lambda}\right)^{s}\right]+\frac{k \lambda}{\alpha}[k(\lambda+\mu)]^{s}\left[1-\left(\frac{k \lambda}{\alpha+k \lambda}\right)^{M-s-1}\right] \\
& +\frac{k}{\alpha+k \lambda}\left(\frac{\lambda+\mu}{\lambda}\right)^{s}(k \lambda)^{M}\left[\frac{1-(k \lambda)^{s}}{1-k \lambda}\right], \\
T_{2}= & \frac{\lambda(1+k)}{(k-1)(\lambda+\mu)}-\frac{k \mu}{(\alpha+k \lambda)(\alpha-k \mu)}\left(1-\left(\frac{k(\lambda+\mu)}{(\alpha+k \lambda)}\right)^{s}\right) \\
& +\frac{k\left(k^{s}-1\right)(\lambda+\mu)^{2}}{(k-1)^{2}(\lambda+\mu+\alpha)(\alpha+k \lambda)}+\frac{\lambda^{2}(\lambda+\mu)}{[\alpha+k \lambda)^{s}(k-1)(\alpha+(k-1) \lambda]}, \\
T_{3}= & \frac{\mu}{\alpha-\mu} \frac{\lambda+\mu}{\lambda+\alpha} \frac{\left(1-((\lambda+\mu) /(\lambda+\alpha))^{s}\right)}{(k-1)(\lambda+\mu+\alpha)}+\frac{\lambda(1+k)+\mu}{(k-1)(\lambda+\mu)}+\frac{\lambda \mu k^{s}(\lambda+\mu)^{s}}{(\lambda+\alpha)(k-1)}, \\
T_{4}= & \frac{\mu(\lambda+\mu)^{2}}{(\alpha-\mu)(\lambda+\alpha)(\lambda+\mu+\alpha)}\left[\frac{k(\lambda+\mu)}{\alpha+k \lambda}\right]^{s} \frac{\alpha+k \lambda}{k(k-1)(\alpha+\lambda)}\left[s-\frac{(\lambda+\mu)^{2}}{(\lambda+\alpha)(\alpha-\mu)}\left(1-\left(\frac{(\lambda+\mu)}{\lambda+\alpha}\right)^{s+1}\right)\right] .
\end{aligned}
$$

4.4. Marginal distributions. Let $Q_{i}^{(1)}$ and $Q_{j}^{(2)}$ denote the marginal distributions of the inventory level and number of waiting customers in the orbit, respectively. 
4.4.1. Distribution of the inventory level. We have $Q_{i}^{(1)}=\sum_{j=0}^{\infty} q(i, j)$. Then from the above set of steady state probabilities we can obtain

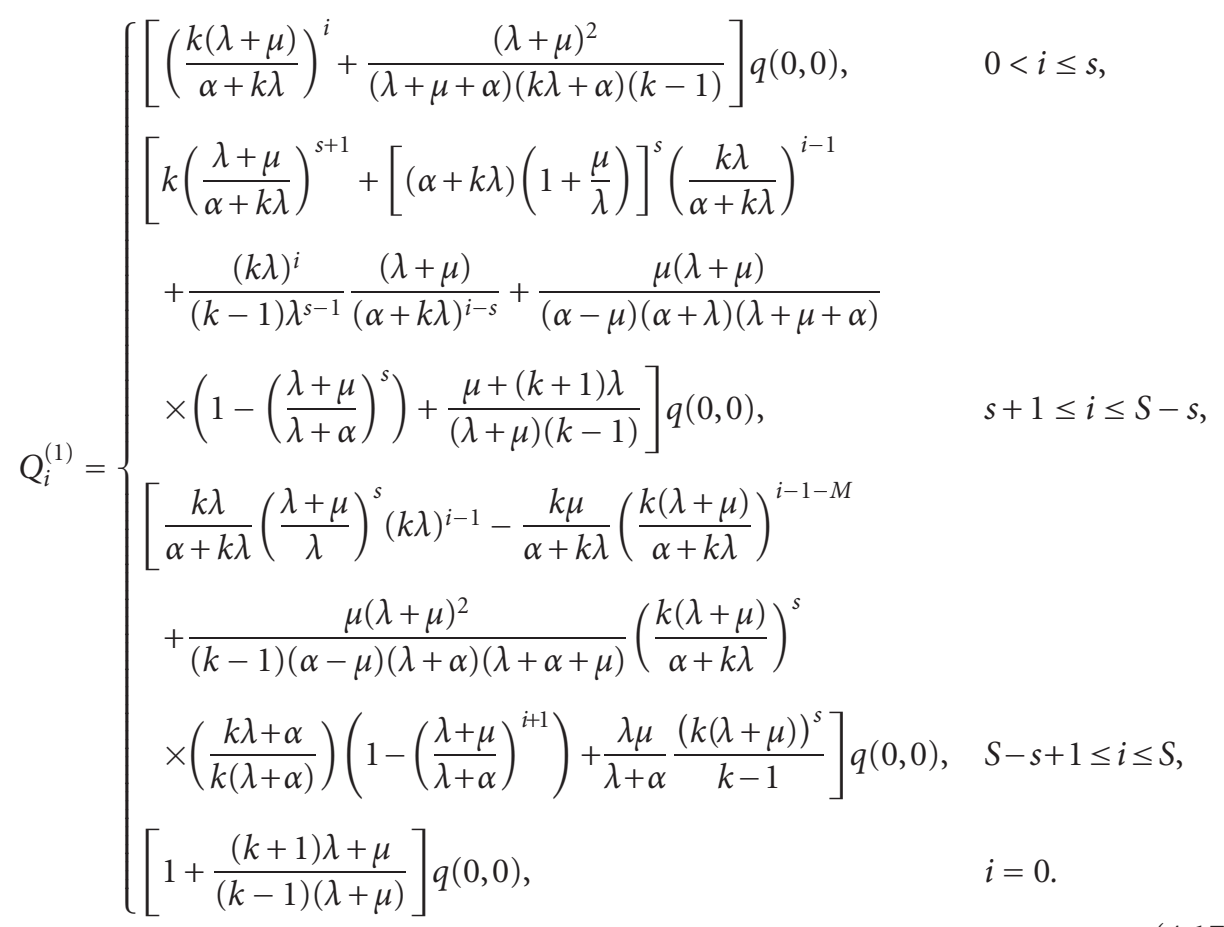

4.4.2. Distribution of the waiting customers. We have $Q_{j}^{(2)}=\sum_{i=0}^{S} q(i, j)$. Then

$$
Q_{j}^{(2)}=\left\{\begin{array}{l}
{\left[\frac{(k+1) \lambda+\mu}{\lambda+\mu}+\frac{k\left(1-k^{s}\right)}{1-k} \frac{(\lambda+\mu)^{2}}{(\lambda+\mu+\alpha)(k \lambda+\alpha)}+\frac{\lambda^{2}(\lambda+\mu)}{\alpha+(k-1) \lambda}\left[1-\left(\frac{\lambda}{\alpha+k \lambda}\right)^{M-s-1}\right]\right.} \\
\quad+\frac{\mu(\lambda+\mu)}{(\alpha-\mu)(\lambda+\mu)(\lambda+\mu+\alpha)}\left(1-\left(\frac{\lambda+\mu}{\lambda+\alpha}\right)^{s}\right)+\frac{(k+1) \lambda+\mu}{\lambda+\mu} \\
\quad+\frac{\mu(\lambda+\mu)^{2}}{(\alpha-\mu)(\lambda+\mu+\alpha)}\left(\frac{k(\lambda+\mu)}{k \lambda+\alpha}\right)^{s} \frac{(k \lambda+\alpha)(\alpha-\mu)}{\lambda+\alpha} \\
\left.\quad \cdot\left[(s-1)(\alpha-\mu)-\left(\frac{\lambda+\mu}{\lambda+\alpha}\right)^{2}\left(1-\left(\frac{\lambda+\mu}{\lambda+\alpha}\right)^{s-1}\right)\right]\right] q(0,0), \\
{\left[1+\frac{\lambda+\mu}{\alpha+(k-1) \lambda+\mu}\left(1-\left(\frac{\lambda+\mu}{\alpha+k \lambda}\right)^{s+1}\right)+[k \lambda(\lambda+\mu)]^{s}\left[1+\frac{k \lambda}{\alpha}\right]\left[1-\left(\frac{k \lambda}{\alpha+k \lambda}\right)^{M-s}\right]\right.} \\
\left.+\frac{(k \lambda)^{s-s+1}}{\alpha+k \lambda}\left(1+\frac{\mu}{\lambda}\right)^{s}\left(\frac{1-(k \lambda)^{s}}{1-k \lambda}\right)-\frac{k \mu}{\alpha-k \mu}\left[1-\left(\frac{k(\lambda+\mu)}{\alpha+k \lambda}\right)^{s}\right]\right] q(0,0), \quad j=0 .
\end{array}\right.
$$


4.5. Expected number of customers in the orbit. Let $E(O)$ denote the expected number of customers in the orbit. Then $E(O)=\sum_{j=1}^{\infty} j Q_{j}^{2}$. Then we can obtain it as

$$
\begin{aligned}
E(O)=\frac{k}{(k-1)^{2}}\{ & \frac{(k+1) \lambda+\mu}{\lambda+\alpha}+\frac{k(1-k)(\lambda+\mu)^{2}}{(1-k)(\lambda+\mu+\alpha)(k \lambda+\alpha)} \\
& +\frac{\lambda^{2}(\lambda+\mu)}{\alpha+(k-1) \lambda}\left[1-\left(\frac{\lambda}{\alpha+k \lambda}\right)^{M-s-1}\right]+\frac{\mu(\lambda+\mu)}{(\alpha-\mu)(\lambda+\alpha)(\lambda+\alpha+\mu)} \\
& \cdot\left(1-\left(\frac{\lambda+\mu}{\lambda+\alpha}\right)^{s}\right)+\frac{(k+1) \lambda+\mu}{\lambda+\mu}+\frac{\mu}{\alpha-\mu} \frac{(\lambda+\mu)^{2}}{(\lambda+\mu+\alpha)}+\left[\frac{(\lambda+\mu) k}{k \lambda+\alpha}\right]^{s} \\
& \left.\times \frac{(k \lambda+\alpha)(\alpha-\mu)}{\lambda+\alpha}\left[(s-1)(\alpha-\mu)-\left(\frac{\lambda+\mu}{\lambda+\alpha}\right)^{2}\left(1-\left(\frac{\lambda+\mu}{\lambda+\alpha}\right)^{s-1}\right)\right]\right\} q(0,0) .
\end{aligned}
$$

4.6. Expected inventory level. Let $E(I)$ denote the expected inventory level in the steady state. Then

$$
E(I)=\sum_{i=1}^{S} i Q_{i}^{1}=\sum_{i=1}^{s} i Q_{i}^{1}+\sum_{i=s+1}^{S-s} i Q_{i}^{1}+\sum_{i=S-s+1}^{S} i Q_{i}^{1} .
$$

Then

$$
\begin{aligned}
E(I)=\{ & \frac{k(\lambda+\mu)(\alpha+k \lambda)}{(\alpha-k \mu)^{2}}\left(1-L_{1}^{s+1}\right)-\frac{s k(\lambda+\mu)}{(\alpha-k \mu)} L_{1}^{s}+\frac{L_{1} s(\lambda+\mu)}{k(k-1)(\lambda+\mu+\alpha)} \\
& +\frac{(\lambda+\mu)(\alpha+k \lambda)^{s}}{(k-1) \lambda^{s}-1} L_{2}\left\{\left(\frac{\alpha+k \lambda}{\alpha}\right)^{2}\left[L_{2}^{s}-L_{2}^{M}\right]+\frac{\alpha+k \lambda}{\alpha}\left[s L_{2}^{s}-M L_{2}^{M}\right]\right\} \\
& +\frac{\mu(M-s)}{(\alpha-\mu)(\lambda+\mu+\alpha)} L_{3}\left(1-L_{3}\right)+(M-s) \frac{(k+1) \lambda+\mu}{(k-1)(\lambda+\mu)} \\
& +L_{2}\left(\frac{\lambda+\mu}{\lambda}\right)^{s}\left[\frac{(k \lambda)^{M}-(k \lambda)^{s}}{(1-k \lambda)^{2}}+\frac{M(k \lambda)^{M}-S(k \lambda)^{S}}{1-k \lambda}\right]-\frac{k \mu}{\alpha+k \lambda}\left[\frac{1-L_{1}^{s}}{\left(1-L_{1}\right)^{2}}+\frac{M-S L_{1}^{s}}{1-L_{1}}\right] \\
& +\frac{\mu(\lambda+\mu)^{2}}{(k-1)(\alpha-\mu)(\lambda+\alpha)(\lambda+\alpha+\mu)} L_{1}^{s} \frac{k \lambda+\alpha}{k(\lambda+\alpha)}\left[s-\frac{\left[L_{3}^{M+2}-L_{3}^{S+2}\right]}{\left(1-L_{3}\right)^{2}}+\frac{M L_{3}^{S}-S L_{3}^{S}}{1-L_{3}}\right] \\
& \left.+\frac{s \lambda \mu}{(\lambda+\alpha)(k-1)}(k(\lambda+\mu))^{s}\right\} q(0,0),
\end{aligned}
$$

where

$$
L_{1}=\frac{k(\lambda+\mu)}{\alpha+k \lambda}, \quad L_{2}=\frac{k \lambda}{\alpha+k \lambda}, \quad L_{3}=\frac{\lambda+\mu}{\lambda+\alpha}
$$




\section{Busy period analysis}

A busy period of the system is defined as the time at which a primary demand is coming to the system when the inventory is full and also none in the orbit and it ends when the inventory is full and none in the orbit. That is, the time to an $(S, 0)$ to $(S, 0)$ transition. To compute this distribution, consider the state $(S, 0)$ as an absorbing state. From the above set of differential difference equations (4.1), remove all the terms corresponding to $(S, 0)$ on the RHS. Then we get

$$
\begin{aligned}
\frac{d}{d t} P(S, j, t)= & -\left(\lambda+j \alpha_{j}\right) P(S, j, t)+\mu P(s, j, t), \quad j \geq 1, \\
\frac{d}{d t} P(i, j, t)= & -\left(\lambda+j \alpha_{j}\right) P(i, j, t)+(j+1) \alpha_{j+1} P(i+1, j+1, t)+\lambda P(i+1, j, t) \\
& +\mu P(i-M, j, t), \quad S-s \leq i \leq S-1, j \geq 1, M=S-s>s, \\
\frac{d}{d t} P(i, j, t)= & -\left(\lambda+j \alpha_{j}\right) P(i, j, t)+(j+1) \alpha_{j+1} P(i+1, j+1, t)+\lambda P(i+1, j, t), \\
& s+1<i \leq S-s-1, j \geq 1, \\
\frac{d}{d t} P(i, j, t)= & -\left(\lambda+j \alpha_{j}+\mu\right) P(i, j, t)+(j+1) \alpha_{j+1} P(i+1, j+1, t)+\lambda P(i+1, j, t),
\end{aligned}
$$

$$
\begin{aligned}
& \frac{d}{d t} P(S-1,0, t)=-\lambda P(S-1,0, t)+\alpha_{1} P(S, 1, t)+\mu P(s-1,0, t), \\
& \frac{d}{d t} P(S, 0, t)=\mu P(s, 0, t), \\
& \frac{d}{d t} P(i, 0, t)=-\lambda P(i, 0, t)+\alpha_{1} P(i+1,1, t)+\lambda P(i+1,0, t)+\mu P(i-M, 0, t), \\
& \quad S-i \leq S-2, \\
& \begin{array}{r}
\frac{d}{d t} P(i, 0, t)=-(\lambda+\mu(1-\delta i)) P(i, 0, t)+\alpha_{1} P(i+1,1, t)+\lambda P(i+1,0, t), \\
0 \leq i \leq S-s-1,
\end{array}
\end{aligned}
$$

where $\delta_{i}=1$ or 0 , according as $i=s+1, s+2, \ldots, S-s-1$ or $i=0,1, \ldots, s$,

$$
\begin{aligned}
\frac{d}{d t} P(0, j, t)= & -(\lambda+\mu) P(0, j, t)+(j+1) \alpha_{j+1} P(1, j+1, t) \\
& +\lambda[P(0, j-1, t)+P(1, j, t)], \quad j \geq 1 .
\end{aligned}
$$


Taking Laplace transforms with respect to $t$ and with the initial condition $P(S, 0,0)=1$ and $P(i, j, 0)=0$ for $i \neq S, j \neq 0$, and solving the equation with $\alpha_{j}=\alpha / j, j \geq 1$, by the above procedure as in Section 4.4, we get

$$
\begin{aligned}
& P(i+1,0, z)=\left[\frac{y_{0}(z)(z+\lambda+\mu)}{\alpha+\lambda y_{0}(z)}\right]^{i+1} P(0,0, z), \quad 0<i \leq s \\
& P(i+1,0, z)=\left[\frac{y_{0}(z)(z+\lambda)}{\alpha+\lambda y_{0}(z)}\right]^{i-s}\left[\frac{y_{0}(z)(z+\lambda+\mu)}{\alpha+\lambda y_{0}(z)}\right]^{s+1} P(0,0, z), \quad s<i \leq s-s-1, \\
& P(i+1,0, z)=\left[\frac{y_{0}(z)(z+\lambda)}{\alpha+\lambda y_{0}(z)}\right]^{i+1-s}\left\{\left[\frac{y_{0}(z)(z+\lambda)}{\alpha+\lambda y_{0}(z)}\right]^{M-s-1}\left(\frac{y_{0}(z)(z+\lambda+\mu)}{\alpha+\lambda y_{0}(z)}\right)^{s+1}\right. \\
& \left.+1-\left(\frac{z+\lambda+\mu}{z+\lambda}\right)^{i+1-M}\right\} P(0,0, z), \quad S-s \leq i \leq S-2, \\
& P(S, 0, z)=\frac{\mu}{z} P(s, 0, z) \\
& P(0, j, z)=\left(1+\frac{\lambda y_{0}(z)}{z+\lambda+\mu}\right)\left(y_{0}(z)^{-j}\right) P(0,0, z), \quad j \geq 1, \\
& P(i, j, z)=\left(y_{0}(z)\right)^{i-j}\left(\frac{z+\lambda+\mu}{z+\lambda+\mu+\alpha}\right)\left(\frac{z+\lambda+\mu}{\alpha+\lambda y_{0}(z)}\right)^{i} P(0,0, z), \quad 0<i \leq s, j \geq 1, \\
& P(i, j, z)=\frac{\left(y_{0}(z)^{-(j+1)}\left(\alpha+\lambda y_{0}(z)\right)\right.}{z+\lambda+\alpha}\left[\frac{y_{0}(z)(z+\lambda)}{\alpha+\lambda y_{0}(z)}\right]^{i-s} \\
& \cdot\left[\frac{y_{0}(z)(z+\lambda+\mu)}{\alpha+\lambda y_{0}(z)}\right]^{s} P(0,0, z), \quad s<i \leq S-s-1, j \geq 1, \\
& P(i, j, z)=\frac{\left(y_{0}(z)^{-(j+1)}\left(\alpha+\lambda y_{0}(z)\right)\right.}{z+\lambda+\alpha}\left(\frac{y_{0}(z)(z+\lambda)}{\alpha+\lambda y_{0}(z)}\right)^{i+1-M} \\
& \left\{\left(\frac{y_{0}(z)(z+\lambda)}{\alpha+\lambda y_{0}(z)}\right)^{M-s-1}\left(\frac{y_{0}(z)(z+\lambda+\mu)}{\alpha+\lambda y_{0}(z)}\right)^{s+1}+\left[1-\left(\frac{z+\lambda+\mu}{z+\lambda}\right)^{i+1-M}\right]\right. \\
& \left.+\mu\left(y_{0}(z)\right)^{-j}\left[\frac{y_{0}(z)(z+\lambda+\mu)}{\alpha+\lambda y_{0}(z)}\right]^{i-M}\right\} P(0,0, z), \\
& S-s \leq i \leq S-1, j \geq 1,
\end{aligned}
$$$$
P(i, j, z)=\frac{\mu}{z+\lambda+\alpha} P(s, j, z), \quad j \geq 1 .
$$ 
$P(0,0, z)$ can be determined by $\sum_{j=0}^{\infty} \sum_{i=0}^{S} P(i, j, z)=1 / z$ which gives $P(0,0, z)=[z H(z)]^{-1}$, where

$$
\begin{aligned}
& \begin{aligned}
H(z)= & h_{4}\left(1-h_{2}^{s+1}\right)-h_{3}\left(1-h_{1}^{s-1}\right)\left[1+h_{1}^{M-s-1} h_{2}^{s+1}\right] \\
& +h_{2}^{s}\left[h_{2} h_{3}\left(1-h_{1}^{M-2}\right)+h_{2} h_{3} h_{5} y_{0}(z)\left(y_{0}(z)-1\right)^{-2}\left(1+\mu z^{-1}-h_{1}^{M-s-1}\right)\right] \\
& +\mu\left(y_{0}(z)-1\right)^{-1} h_{7}-h_{4}\left(1-h_{2}^{s-1}\left(1-h_{6}\right)\right)+h_{3} h_{5} y_{0}(z)\left(y_{0}(z)-1\right)^{-1} \\
& \cdot\left(1-h_{1}^{s}\right)\left(1+h_{1}^{M-s-1} h_{2}^{s+1}\right)\left(1-h_{2}^{s}\right) h_{8},
\end{aligned} \\
& h_{1}=\frac{y_{0}(z)(z+\lambda)}{\alpha+\lambda y_{0}(z)}, \quad h_{2}=\frac{y_{0}(z)(z+\lambda+\mu)}{\alpha+\lambda y_{0}(z)}, \quad h_{3}=\frac{y_{0}(z)(z+\lambda)}{\alpha-z y_{0}(z)}, \\
& h_{4}=\frac{y_{0}(z)(z+\lambda+\mu)}{\alpha-(\mu+z) y_{0}(z)}, \quad h_{5}=\frac{\alpha+\lambda y_{0}(z)}{z+\lambda+\alpha}, \quad h_{6}=\frac{z+\lambda+\mu}{\left(y_{0}(z)-1\right)(z+\lambda+\mu+\alpha)}, \\
& h_{7}=\frac{z+\lambda+\mu}{(z+\lambda+\alpha)^{2}}, \quad h_{8}=\mu\left(y_{0}(z)-1\right)^{-1}\left(\alpha-y_{0}(z)\right)(z+\mu)^{-1}-h_{4} h_{5}\left(y_{0}(z)\right)^{-1} .
\end{aligned}
$$

5.1. Expected length of the busy period. We have

$$
P(S, 0, z)=\frac{\mu}{z} P(s, 0, z)=\frac{\mu}{z} h_{2}^{s} P(0,0, z) .
$$

$P(S, 0, z)$ is the cumulative distribution function of the Laplace transform of the busy period. Define $f(S, 0, t) d t=P$ \{busy period ends in $(t, t+d t)$ leaving back the inventory level to $S$ and " 0 " demands in the orbit for the first time $\}$.

Hence $f(S, 0, t)=(d / d t) P(S, 0, t)$ is the pdf of $P(S, 0, t)$. Let $f(S, 0, z)$ be the Laplace transform of the busy period density $f(S, 0, t)$. Then

$$
f(S, 0, z)=z P(S, 0, z)=\mu h_{2}^{s} P(0,0, z) .
$$

Then the expected amount of time, the system is continuously busy, is

$$
E(T)=-\left[\frac{d}{d z} f(S, 0, z)\right]_{z=0}
$$

which gives

$$
\begin{gathered}
E(T)=-\mu\left\{\frac{k+(\lambda+\mu)(1-k \lambda) V}{\alpha+k \lambda} \frac{(k-1)^{2} \alpha(\lambda+\alpha) s}{\lambda \mu(\lambda+\mu) k^{3}}\left(\frac{k(\lambda+\mu)}{\alpha+k \lambda}\right)^{s-1}\right. \\
\left.-\left(\frac{k(\lambda+\mu)}{\alpha+k \lambda}\right)^{s}\left(\frac{(k-1)^{2} \alpha(\lambda+\alpha)}{\lambda \mu(\lambda+\mu) k^{3}}\right)^{2}\left(H_{0}+H_{0}^{\prime}\right)\right\},
\end{gathered}
$$


where

$$
\begin{aligned}
k= & \lim _{z \rightarrow 0} y_{0}(z), \quad V=\lim _{z \rightarrow 0} y_{0}^{\prime}(z), \quad H_{0}=\lim _{z \rightarrow 0} H(z), \quad H_{0}^{\prime}=\lim _{z \rightarrow 0} H^{\prime}(z), \quad h_{i 0}=\lim _{z \rightarrow 0} h_{i}, \\
H_{0}= & h_{40}\left(1-h_{2}^{s+1}\right)+h_{20}^{s+1} h_{30}\left(1-h_{10}^{M-s-1}\right)+h_{10}^{M-s-1} h_{20}^{s+1} h_{30}\left(1-h_{10}^{s-1}\right) \\
& +h_{50} h_{20}^{s+1} \frac{k \lambda}{(k-1) \alpha}\left(1-h_{10}^{M-s-1}\right)+h_{50}\left[h_{10}^{M-s-1} h_{20}^{s+1} \frac{\lambda}{\alpha(k-1)}\left(1-h_{10}^{s}\right)\right] \\
& +h_{50} \frac{\lambda}{\alpha(k-1)}\left(1-h_{10}^{s}\right)-h_{50} h_{40}\left(1-h_{20}^{s}\right) \frac{1}{k(k-1)}+\frac{\mu}{(k-1)(\alpha-k \mu)}\left(1-h_{20}^{s}\right) \\
& +\frac{\mu}{\lambda+\alpha} \frac{\lambda+\mu}{\lambda+\alpha} \frac{h_{20}}{k}, \\
H_{0}^{\prime}= & h_{30} h_{50} V\left\{\frac{k}{(k-1)^{2}}(s+1) h_{2}^{s}\left[\frac{(\alpha+k \lambda)(\lambda+\mu+k)-k(\lambda+\mu) \lambda}{(\alpha+k \lambda)^{2}}\right]-\left(k^{2}-1\right)\right\} .
\end{aligned}
$$

5.2. Expected number of primary demands during a lead time. Let $r_{n, k}$ be the probability that there are $n$ primary and $k$ repeated demands during a lead time. Then

$$
\begin{aligned}
r_{n, k} & =\int_{0}^{\infty} e^{-}(\lambda+\alpha+\mu) x \frac{(\lambda x)^{n}}{n !} \frac{(\alpha x)^{k}}{k !} \mu e^{-\mu x} d x \\
& =\left(\begin{array}{c}
n+k \\
k
\end{array}\right)\left(\frac{\alpha}{\alpha+\lambda+\mu}\right)^{k}\left(\frac{\lambda}{\alpha+\lambda+\mu}\right)^{n} \frac{\mu}{\alpha+\lambda+\mu}, \quad n, k \geq 0 .
\end{aligned}
$$

Therefore, expected number of primary demands during a lead time when $k$ demands in the orbit

$$
\begin{aligned}
& =\sum_{n=1}^{\infty} n r_{n, k} \\
& =\left(\frac{\alpha}{\alpha+\lambda+\mu}\right)^{k}\left(\frac{\mu}{\alpha+\lambda+\mu}\right) \sum_{n=1}^{\infty} n\left(\begin{array}{c}
n+k \\
k
\end{array}\right)\left(\frac{\lambda}{\alpha+\lambda+\mu}\right)^{n} .
\end{aligned}
$$

5.3. Expected number of secondary demands during a lead time. Expected number of secondary demands during a lead time when there are $n$ primary calls

$$
\begin{aligned}
& =\sum_{k=1}^{\infty} k r_{n, k} \\
& =\left(\frac{\lambda}{\lambda+\alpha+\mu}\right)^{n} \frac{\mu}{\lambda+\alpha+\mu} \sum_{k=1}^{\infty} k\left(\begin{array}{c}
n+k \\
k
\end{array}\right)\left(\frac{\alpha}{\lambda+\alpha+\mu}\right)^{k} .
\end{aligned}
$$

5.4. Distribution of the inventory dry period. During the lead time the inventory level may come down to zero and again replenishment may not take place for a random amount of time. This period is the inventory dry period. The time between the consecutive replenishment is distributed as exponential with parameter $\mu$ and the dry period 
is the residual of the replenishment time which is again distributed as exponential with parameter $\mu$.

\section{Waiting time}

Suppose that at time $t$, a customer places a primary request. Then the period until that request starts to be served is its waiting time.

Assume that at time $t=0$, there are $j$ customers in orbit and the inventory level is $i, i=0,1, \ldots, S, j \geq 1$. Tag one of the customers in the orbit and denote by $f_{i j}(t)$, the probability that by time $t$ this customer has not been served. That is, the residual waiting time $\tau_{i j}$ of the tagged customer is greater than $t$. Then

$$
f_{i j}(t)=P\left\{\tau_{i j} \geq t\right\}, \quad i=0,1, \ldots, S, j \geq 1
$$

To get equations for the probabilities $f_{i j}(t)$, we introduce an auxiliary Markov process $\xi(t)$ with state space $\{0,1,2, \ldots, S\} \times\{1,2, \ldots\} \cup\{a\}$. The state $(i, j)$ can be thought of as the presence in the system of $i$ units in the inventory and $j$ customers in the orbit including the tagged one. The special state $a$ is an absorbing state and the transition into this state means that the tagged customer is served. Thus the residual waiting time of the tagged customer, $\tau_{i j}$, is the time till absorption to state $a$. Then

$$
\begin{aligned}
f_{i j}(t) & =P\{\xi(t) \neq a / \xi(0)=(i, j)\} \\
& =1-P\{\xi(t)=a / \xi(0)=(i, j)\} .
\end{aligned}
$$

The transitions among the various states of $\{\xi(t)\}$ are shown in the following diagram. From state $(i, j)$ the process can move to any of the following states:

(i) for $s<i \leq S, j \geq 1$,

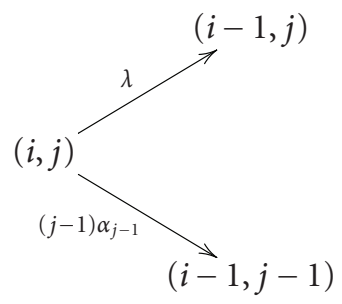

(ii) for $0<i \leq s, j \geq 1$,

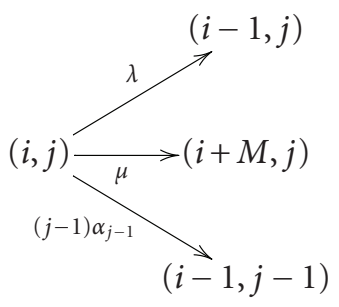


(iii) for $i=0, j \geq 1$,



Therefore from the Kolmogorov backward equation for the Markov process $\{\xi(t)\}$, we get

$$
\begin{array}{lr}
\frac{d}{d t} f_{i j}(t)=-\left(\lambda+j \alpha_{j}\right) f_{i j}(t)+\lambda f_{i-1 j}(t)+(j-1) \alpha_{j-1}(t), \quad s<i \leq S, j \geq 1, \\
\frac{d}{d t} f_{i j}(t)=-\left(\lambda+\mu+j \alpha_{j}\right) f_{i j}(t)+\lambda f_{i-1 j}(t)+(j-1) \alpha_{j-1} f_{i-1 j-1}(t)+\mu f_{i+M, j}(t), \\
0<i \leq s, j \geq 1, \\
\frac{d}{d t} f_{0 j}(t)=-\left(\lambda+\mu+j \alpha_{j}\right) f_{0 j}(t)+\lambda f_{0 j+1}(t)+\mu f_{M, j}(t), \quad j \geq 1 .
\end{array}
$$

To solve this system, the initial condition is

$$
f_{i j}(0)=1, \quad 0 \leq i \leq S, j \geq 1 \text {. }
$$

Define the Laplace transform $\phi_{i j}(z)=\int_{0}^{\infty} e^{-z t} f_{i j}(t) d t,|z| \leq 1$. From (6.6) we have

$$
\begin{array}{rr}
z \phi_{i j}(z)-1=-\left(\lambda+j \alpha_{j}\right) \phi_{i j}(z)+\lambda \phi_{i-1 j}(z)+(j-1) \alpha_{j-1} \phi_{i-1 j-1}(z), & s \leq i \leq S, j \geq 1, \\
z \phi_{i j}(z)-1=-\left(\lambda+j \alpha_{j}+\mu\right) \phi_{i j}(z)+\lambda \phi_{i-1 j}(z)+(j-1) \alpha_{j-1} \phi_{i-1 j-1}(z)+\mu \phi_{i+M, j}(z), & 0<i \leq s, j \geq 1, \\
z \phi_{0 j}(z)-1=-\left(\lambda+j \alpha_{j}+\mu\right) \phi_{0 j}(z)+\lambda \phi_{0 j+1}(z)+\mu \phi_{M, j}(z), \quad j \geq 1 .
\end{array}
$$

We will solve this system of nonhomogeneous equations as difference equation in $i$ and $j$. Set $\alpha_{j}=\alpha / j$ and

$$
\phi_{i-1 j}(z)=y^{(-i)} \phi_{0 j}(z)=y^{-(i+j)} \phi_{01}(z), \quad(i, j) \in E .
$$

Equation (6.11) to be a solution to (6.9), (6.9) should satisfy the following.

From (6.9), the homogeneous part gives $\left[(z+\lambda+\mu+\alpha)-\lambda y-\alpha y^{2}-\mu y^{-M}\right] \phi_{01}(z)=$ 0 , which gives

$$
z+\lambda+\mu+\alpha-\lambda y-\alpha y^{2}-\mu y^{-M}=0 .
$$


By Rouche's theorem (6.12) is having $M+1$ roots of which $M$ roots inside and one root outside the unit circle $|y|=1$. Consider the outside root, say $y_{0}(z)$. Therefore

$$
\phi_{i j}(z)=\left(y_{0}(z)\right)^{-(i+j)} \phi_{01}(z), \quad 0<i \leq s, j \geq 1
$$

and the solution of the particular function is $1 / z$. Therefore the complete solution is

$$
\phi_{i j}(z)=\left(y_{0}(z)\right)^{-(i+j)} \phi_{01}(z)+\frac{1}{z}, \quad 0<i \leq s, j \geq 1 .
$$

Using this we can recursively compute the remaining Laplace transforms. Therefore from (6.8),

$$
\phi_{i j}(z)=\left(\frac{\lambda+\alpha y_{0}(z)}{z+\alpha+\alpha}\right)^{i-s}\left(y_{0}(z)\right)^{-(s+j+1)} \phi_{01}(z)+\frac{1}{z}, \quad s<i \leq S, j \geq 1 .
$$

From (6.9),

$$
\begin{aligned}
\lambda \phi_{0 j}(z) & +\alpha \phi_{0 j-1}(z) \\
= & {\left[(z+\lambda+\mu+\alpha) y_{0}(z)^{-(j+1)}-\mu\left(\frac{\lambda+\alpha y_{0}(z)}{z+\lambda+\alpha}\right)\left(y_{0}(z)\right)^{-(s+j+1)}\right] \phi_{01}(z)-1, \quad j \geq 1 . }
\end{aligned}
$$

From (6.10) and (6.11),

$$
\begin{aligned}
\phi_{0 j}(z)= & {\left[\left(\frac{z+\lambda+\mu+\alpha}{\lambda}\right)\left(y_{0}(z)\right)^{-(j+1)}-\frac{\mu}{\lambda}\left(\frac{\lambda+\alpha y_{0}(z)}{z+\lambda+\alpha}\right)^{(M-s+1)}\left(y_{0}(z)\right)^{-(s+j+1)}\right] \phi_{01}(z) } \\
& -\frac{\alpha}{\lambda} \phi_{0 j-1}(z)-\frac{1}{\lambda}, \quad j \geq 2 .
\end{aligned}
$$

Iteratively,

$$
\begin{aligned}
\phi_{0 j}(z)= & {\left[C_{j}(z)-\left(\frac{\alpha}{\lambda}\right) C_{j-1}(z)+\left(\frac{\alpha}{\lambda}\right)^{2} C_{j-2}(z)-\cdots+(-1)^{j-1}\left(\frac{\alpha}{\lambda}\right)^{j-1} C_{1}(z)+(-1)^{j}\left(\frac{\alpha}{\lambda}\right)^{j} C_{0}(z)\right] } \\
& \times \phi_{01}(z)-\frac{1}{\lambda}\left[1-\left(\frac{\alpha}{\lambda}\right)+\left(\frac{\alpha}{\lambda}\right)^{2}-\left(\frac{\alpha}{\lambda}\right)^{3}+\cdots+(-1)^{j}\left(\frac{\alpha}{\lambda}\right)^{j-2}\right], \quad j \geq 3, \\
\phi_{02}(z)= & {\left[C_{2}(z)-\left(\frac{\alpha}{\lambda}\right) C_{1}(z)+\left(\frac{\alpha}{\lambda}\right)^{2}\right] \phi_{01}(z)-\frac{1}{\lambda}, }
\end{aligned}
$$


where

$$
C_{j}(z)=\frac{z+\lambda+\mu+\alpha}{\lambda}\left(y_{0}(z)\right)^{-(j+1)}-\frac{\mu}{\lambda}\left(\frac{\lambda+\alpha y_{0}(z)}{z+\lambda+\alpha}\right)^{M-s+1}\left(y_{0}(z)\right)^{-(s+j+1)},
$$

$$
j \geq 1 \text { with } C_{0}(z)=1 .
$$

$\phi_{01}(z)$ can be obtained in such a way that $\sum_{j=1}^{\infty} \sum_{i=0}^{S} \phi_{i j}(z)=1 / z$ which gives

$$
\phi_{01}(z)=\left(y_{0}(z)\right)^{S-1} \frac{\left[1-y_{0}(z)\right]^{2}}{\left[1-\left(y_{0}(z)\right)^{S+1}\right]}
$$

6.1. Conditional mean waiting times. By taking limit as $z \rightarrow 0$, we get the mean conditional waiting times. Let $m_{i j}=\lim _{z \rightarrow 0} \phi_{i j}(z)$. Set $\lim _{z \rightarrow 0} y_{0}(z)=k_{1}$ and $C_{j 0}=\lim _{z \rightarrow 0} C_{j}(z)$. Then from (6.12), we have

$$
(\lambda+\mu+\alpha)-\lambda k_{1}-\alpha k_{1}^{2}-\mu k_{1}^{-M}=0
$$

and $k_{1}$ is the root of $(6.21)$. Then we have

$$
\begin{aligned}
& m_{i j}=k_{1}^{-(i+j)} m_{01}, \quad 0<i \leq s, j \geq 1, \\
& m_{i j}=\left(\frac{\lambda+\alpha k_{1}}{\lambda+\alpha}\right)^{i-s} k_{1}^{-(s+j+1)} m_{01}, \quad s<i \leq S, j \geq 1, \\
& m_{0 j}=\sum_{i=0}^{j}(-1)^{i}\left(\frac{\alpha}{\lambda}\right)^{i} C_{j-i} m_{01}-\frac{1}{\lambda} \sum_{i=0}^{j-1}(-1)^{i}\left(\frac{\alpha}{\lambda}\right)^{i}, \quad j \geq 2, \\
& m_{01}=\frac{k_{1}^{S-1}\left[1-k_{1}\right]^{2}}{1-k_{1}^{S+1}} .
\end{aligned}
$$

6.2. Unconditional mean waiting times. Expected waiting time of a unit in the steady state is given by

$$
E(W)=\sum_{i=0}^{S} \sum_{j=1}^{\infty} m_{i j} q(i, j)=m_{01} q(0,1)+\sum_{j=2}^{\infty} m_{0 j} q(0, j)+\sum_{i=1}^{S} \sum_{j=1}^{\infty} m_{i j} q(i, j) .
$$


Then, let

$$
\begin{gathered}
\theta_{1}=\frac{\lambda+\alpha k_{1}}{\lambda+\alpha}, \quad \theta_{2}=\frac{\lambda+\mu+k \lambda}{\lambda+\mu}, \quad \theta_{3}=\frac{\lambda+\mu}{\lambda+\alpha}, \quad \theta_{4}=\frac{\lambda+\alpha k_{1}}{\lambda+\mu}, \\
\theta_{51}=\frac{\alpha^{2}}{\lambda^{2}-\alpha^{2}}, \quad \theta_{5}=\theta_{51}\left(\frac{\alpha}{\lambda}\right)^{2}+\frac{\alpha^{2}}{\lambda(\lambda+k \alpha)}, \quad \theta_{6}=\frac{k(\lambda+\mu)}{\alpha+k \lambda}, \quad \theta_{7}=\frac{\lambda+\mu}{\lambda+\mu+\alpha} .
\end{gathered}
$$

Then, we get

$$
\begin{aligned}
E(W)=\{ & \theta_{2}+\frac{\lambda+\mu+\alpha}{\lambda k_{1}\left(k_{1}^{2}-1\right)}-\theta_{5}-\frac{k_{1}^{-s}\left(1-k_{1}\right) \mu \theta_{1}^{M-s+1}}{\lambda+\alpha k_{1}}\left[\frac{1}{k_{1}\left(k_{1}^{2}-1\right)}+\frac{\alpha}{\lambda} \theta_{51}\right] \\
& +\left[\left(k^{2}-1\right)^{-1}+\left(k^{3}-1\right)^{-1}-\left(\frac{\alpha}{k}\right)^{2} \frac{k-1}{\lambda(k \lambda-\alpha)}\right] \frac{1}{m_{01}(\lambda+\alpha)} \\
& +\frac{\theta_{6} \theta_{7}\left(1-k_{1}^{-s}\right)}{k(\lambda+\mu+\alpha) k_{1}\left(1-k_{1}\right)\left(k k_{1}-1\right)}+\frac{k^{s+1} \theta_{1} \theta_{3} \lambda}{k_{1}^{s+1}\left(k k_{1}-1\right)}\left(1-\frac{k \lambda \theta_{1}}{k \lambda+\alpha}\right)^{M-s-1} \\
& +\frac{\mu \theta_{3}\left(1-\theta_{3}^{s}\right)}{\alpha-\mu}+\frac{k_{1}^{-(s+1)}}{k_{1}-1} \theta_{1}^{M-s} \theta_{2} \\
& +\frac{\mu}{\alpha-\mu} \theta_{3} \theta_{6}^{s} \theta_{7}\left[\frac{\left(k_{1} \theta_{1}\right)^{-s}}{k_{1}-1} \frac{\lambda+\alpha}{\alpha\left(1-k_{1}\right)} \cdot \theta_{1}^{M+1}\left(1-\theta_{1}^{s-1}\right)-\frac{(\lambda+\alpha)^{-(s+1)}}{\mu-\alpha k_{1}} \theta_{3} \theta_{1}^{M+1}\left(1-\theta_{4}^{s-1}\right)\right] \\
& \left.+\frac{\lambda \mu}{\lambda+\alpha} k^{s} \theta_{1}^{M} \frac{k_{1}^{-(s+1)}}{k k_{1}-1}\right\} m_{01} q(0,0) .
\end{aligned}
$$

\section{Control problem}

In this section we investigate the optimal values of the control parameters " $s$ " and "S." For this, we formulated a cost function which is to be minimized. The following main costs are considered:

(i) fixed setup cost " $K$ ” per unit time;

(ii) the ordering cost " $C$ " per unit time;

(iii) the waiting time cost $\omega$ per unit time due to waiting in the orbit.

Let $T(s, S)$ be the total expected cost per unit time which is a function of the control variates $s$ and $S$. Then we define $T(s, S)$ as $T(s, S)=K+C$ (expected number of reorders per unit time) $+\omega$ (expected waiting time in the orbit).

To obtain the optimal values of " $s$ " and " $S$," we will have to examine the convexity properties of $T(s, S)$. To do this, we have to obtain the expected number of reorders per unit time. The expected number of reorders in a cycle starting from $(S, 0)$ before returning to $(S, 0)$ for the first time is the same as the expected number of visits to state $(s, j)$, $j \geq 0$, starting from $(S, 0)$ before returning to $(S, 0)$ for the first time. In each visit to $(s, j)$, $j \geq 0$, a replenishment order will be placed and only one order is pending at any given 
Table 7.1. The total expected cost per unit time for $\lambda=0.1, \alpha=0.02, \mu=0.5, s=1, K=100, C=10$, $\omega=5$.

\begin{tabular}{l|cccccc}
\hline$S$ & 10 & 11 & 12 & 13 & 14 & 15 \\
\hline$T(S, s)$ & 100.0119 & 100.0117 & 100.0116 & 100.0115 & 100.0115 & 100.0116 \\
\hline$S$ & 16 & 17 & 18 & 19 & 20 & - \\
\hline$T(S, s)$ & 100.0118 & 100.0122 & 100.0128 & 100.0136 & 100.0148 & - \\
\hline
\end{tabular}

Table 7.2. The total expected cost per unit time for $\lambda=0.01, \alpha=2.5, \mu=6, S=40, K=100, C=10$, $\omega=0.5$.

\begin{tabular}{l|ccccccc}
\hline$s$ & 2 & 3 & 4 & 5 & 6 & 7 & 8 \\
\hline$T(S, s)$ & 100.0071 & 28.0026 & 4.3492 & 88.3344 & 99.3664 & 99.9749 & 99.9991 \\
\hline$s$ & 9 & 10 & - & - & - & - & - \\
\hline$T(S, s)$ & 99.9999 & 100.0000 & - & - & - & - & - \\
\hline
\end{tabular}

time. From the theory of regenerative process, the stationary probability $q(i, j)$ can be interpreted as the long run fraction of time the system is in state $(i, j)$. Then the expected number of replenishments per unit time is the expected number of visits to state $(s, j)$, $j \geq 0$, and can be computed as $\sum_{j=0}^{\infty}(\lambda+j \alpha) q(s+1, j)$. Now, $\sum_{j=0}^{\infty}(\lambda+j \alpha) q(s+1, j)=$ $\left.\left(k^{s+1}(\lambda+\mu) /(\alpha+k \lambda)\right)(\lambda /(k-1))^{2}(\lambda(k-1)+\alpha)+\lambda[k(\lambda+\mu)]^{s}\right] q(0,0)$, using the stationary probabilities given in Section 4.3. The expected waiting time in the orbit is given in Section 6.2. Then the total expected cost per unit time is

$$
T(s, S)=K+C\left[\frac{k^{s+1}(\lambda+\mu)}{\alpha+k \lambda}\left(\frac{\lambda}{k-1}\right)^{2}(\lambda(k-1)+\alpha)+\lambda[k(\lambda+\mu)]^{s}\right] q(0,0)+\omega E(W) .
$$

THEOREM 7.1. The function $T(s, S)$ given in (7.1) is convex in $S$ for a fixed s and is convex in $s$ for a fixed $S$ and hence the global minimum $S *$ of $S$ exists for fixed s and global minimum $s *$ of $s$ exists for fixed $S$.

Proof. From (7.1) one can verify that the partial derivatives $\partial^{2} T(s, S) / \partial S^{2} \geq 0, \partial^{2} T(s, S) /$ $\partial s^{2} \geq 0$ and hence the function is convex, respectively, in $S$ for fixed $s$ and in $s$ for fixed $S$. Hence the global minimum $S *$ of $S$ and $s *$ of $s$ exists, respectively, for fixed values of $s$ and $S$.

7.1. Numerical results. In this section we discuss some numerical examples that reveal the convexity of the total expected cost function. We performed the calculations using the MATLAB. Table 7.1 gives the total expected cost per unit time for various values of $S$ and fixed values of other parameters and costs. Table 7.2 gives the total expected cost per unit time for various values of $s$ and fixed values of other parameters and costs. From Tables 7.1 and 7.2 it can be seen that the optimal values $S *$ and $s *$ are 13 and 4, respectively. 
Table 7.3. Expected waiting time for a unit in the orbit when $\lambda=0.1, \alpha=0.02, \mu=0.5, s=1$.

\begin{tabular}{l|ccccc}
\hline$S$ & 10 & 11 & 12 & 13 & 14 \\
\hline$E(W)$ & 0.0024 & 0.0024 & 0.0023 & 0.0023 & 0.0023 \\
\hline$S$ & 15 & 16 & 17 & 18 & 19 \\
\hline$E(W)$ & 0.0024 & 0.0025 & 0.0026 & 0.0027 & 0.0030 \\
\hline
\end{tabular}

Table 7.4. Expected waiting time for a unit in the orbit when $\lambda=0.01, \alpha=2.5, \mu=6.0, S=40$.

\begin{tabular}{l|ccccc}
\hline$s$ & 2 & 3 & 4 & 5 & 6 \\
\hline$E(W)$ & 0.0148 & -143.9 & -191.3 & -23.3 & -1.26 \\
\hline$s$ & 7 & 8 & 9 & 10 & - \\
\hline$E(W)$ & -0.0501 & -0.0017 & $-5.5 e^{-5}$ & $-1.3 e^{-6}$ & - \\
\hline
\end{tabular}

Table 7.5. Expected inventory level in the steady state for $s=5, S=20, \mu=5$.

\begin{tabular}{l|cccc}
\hline$\lambda \mid \alpha$ & 0.1 & 0.2 & 0.3 & 0.4 \\
\hline 1.0 & $6.5 e^{15}$ & $1.2 e^{15}$ & $2.4 e^{14}$ & $5.7 e^{13}$ \\
\hline 2.0 & $1.6 e^{12}$ & $6.7 e^{11}$ & $3.1 e^{11}$ & $1.6 e^{11}$ \\
\hline 3.0 & $-8.2 e^{8}$ & $-5.5 e^{8}$ & $-4.1 e^{8}$ & $-3.5 e^{8}$ \\
\hline 4.0 & $-6.0 e^{9}$ & $-6.1 e^{9}$ & $-6.2 e^{9}$ & $-6.2 e^{9}$ \\
\hline 4.5 & $-1.7 e^{15}$ & $-1.5 e^{10}$ & $-1.2 e^{10}$ & $-7.7 e^{9}$ \\
\hline
\end{tabular}

Tables 7.3, 7.4, and 7.5 give average waiting time for various values of $S$ and $s$ and the average inventory level for various values of $\lambda$ and $\alpha$.

\section{Acknowledgment}

The author would like to express her sincere thanks to the unknown referee for the helpful comments and suggestions which help to improve the presentation of the paper.

\section{References}

[1] J. R. Artalejo, A. Krishnamoorthy, and M. J. Lopez-Herrero, Numerical analysis of $(s, S)$ inventory systems with repeated attempts, Annals of Operations Research 141 (2006), no. 1, 67-83.

[2] G. I. Falin and J. G. C. Templeton, Retrial Queues, Chapman and Hall, London, 1997.

[3] S. Kalpakam and G. Arivarignan, Inventory system with random supply quantity, OR Spektrum 12 (1990), no. 3, 139-145.

[4] S. Kalpakam and K. P. Sapna, Continuous review $(s, S)$ inventory system with random lifetimes and positive leadtimes, Operations Research Letters 16 (1994), no. 2, 115-119.

[5] _ An $(s, S)$ perishable system with arbitrarily distributed leadtimes, Operations Research 33 (1996), no. 1, 1-19.

[6] A. Krishnamoorthy and N. Raju, $(s, S)$ inventory systems with random lead time-the n-policy, Korean Journal of Applied Mathematics (1997). 
[7] A. Krishnamoorthy and P. V. Ushakumari, Reliability of a $k$-out-of-n system with repair and retrial of failed units, Top 7 (1999), no. 2, 293-304.

[8] L. Liu and T. Yang, An $(s, S)$ random life time inventory model with a positive lead time, European Journal of Operational Research 113 (1999), no. 1, 52-63.

[9] E. Mohebbi and M. J. M. Posner, A continuous-review inventory system with lost sales and variable lead time, Naval Research Logistics 45 (1998), no. 3, 259-278.

[10] S. Nahmias, Perishable inventory theory: a review, Operations Research 30 (1982), 680-708.

[11] F. Raffat, Survey of literature on continuously deteriorating inventory models, Journal of Operational Research Society 42 (1991), no. 1, 27-37.

[12] R. L. Tweedie, Sufficient conditions for regularity, recurrence, and ergodicity of Markov processes, Mathematical Proceedings of the Cambridge Philosophical Society 78 (1975), part 1, 125-136.

P. V. Ushakumari: Department of Mathematics, Amrita Vishwa Vidyapeetham, Amritapuri, Kollam, Kerala 690 525, India

E-mail address: usha@amritapuri.amrita.edu 




Advances in

Operations Research

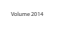

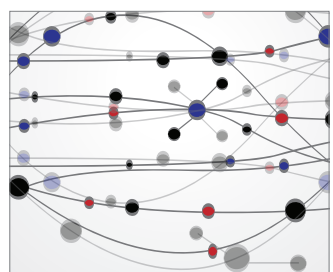

\section{The Scientific} World Journal
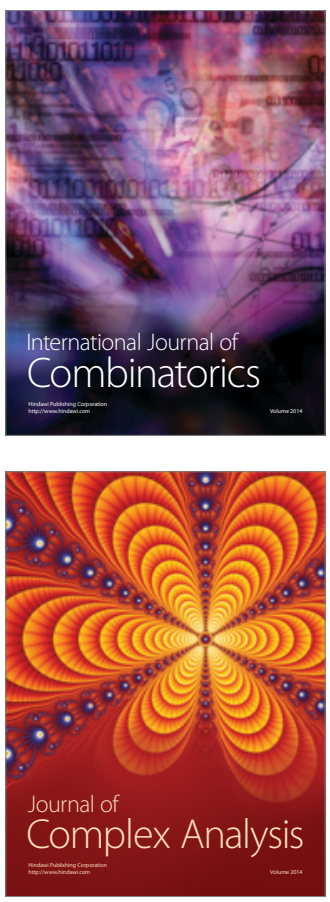

International Journal of

Mathematics and

Mathematical

Sciences
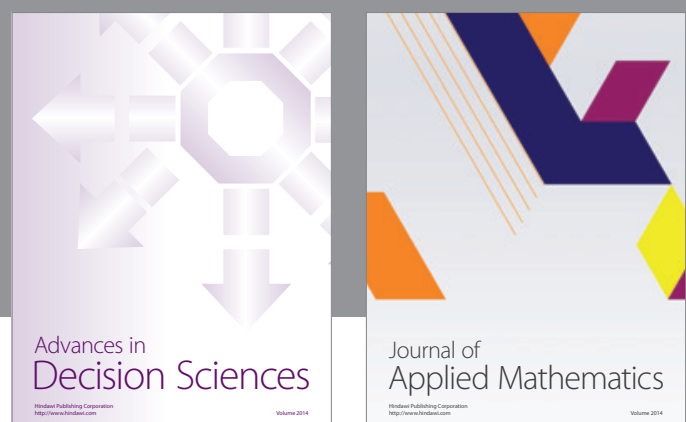

Journal of

Applied Mathematics
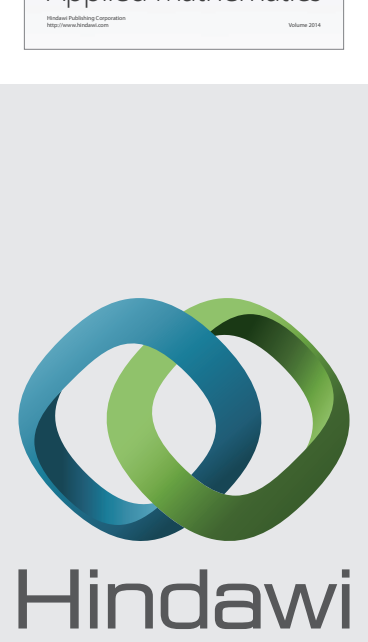

Submit your manuscripts at http://www.hindawi.com
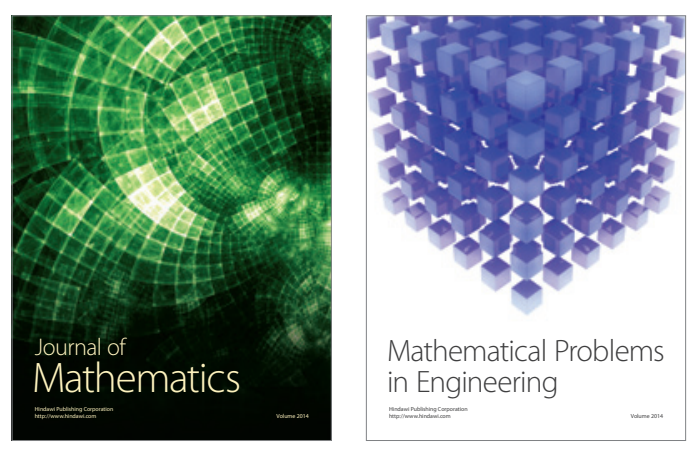

Mathematical Problems in Engineering
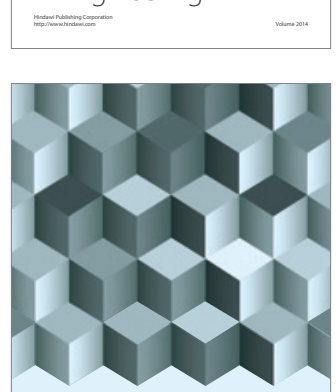

Journal of

Function Spaces


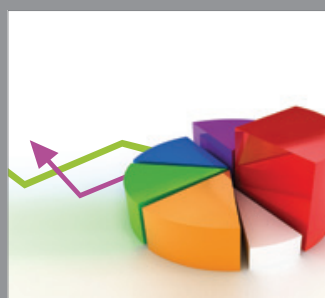

ournal of

Probability and Statistics

Promensencen
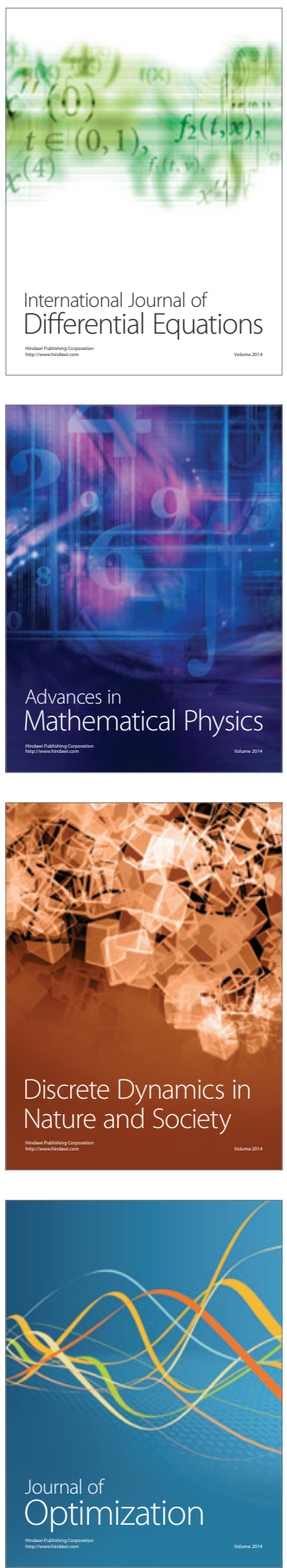